\title{
Enhancing Sideband Cooling by Feedback-Controlled Light
}

\author{
Massimiliano Rossi, ${ }^{1,2}$ Nenad Kralj, ${ }^{2}$ Stefano Zippilli, ${ }^{2,3}$ Riccardo Natali, ${ }^{2,3}$ Antonio Borrielli, ${ }^{4}$ \\ Gregory Pandraud, ${ }^{5}$ Enrico Serra, ${ }^{5,6}$ Giovanni Di Giuseppe, ${ }^{2,3,}$ and David Vitali ${ }^{2,3,7, \dagger}$ \\ ${ }^{1}$ School of Higher Studies "C. Urbani”, University of Camerino, 62032 Camerino (MC), Italy \\ ${ }^{2}$ School of Science and Technology, Physics Division, University of Camerino, 62032 Camerino (MC), Italy \\ ${ }^{3}$ INFN, Sezione di Perugia, 06123 Perugia $(P G)$, Italy \\ ${ }^{4}$ Institute of Materials for Electronics and Magnetism, Nanoscience-Trento-FBK Division, 38123 Povo (TN), Italy \\ ${ }^{5}$ Delft University of Technology, Else Kooi Laboratory, 2628 Delft, Netherlands \\ ${ }^{6}$ Istituto Nazionale di Fisica Nucleare, TIFPA, 38123 Povo (TN), Italy \\ ${ }^{7}$ CNR-INO, L.go Enrico Fermi 6, I-50125 Firenze, Italy \\ (Received 13 April 2017; published 22 September 2017)
}

\begin{abstract}
We realize a phase-sensitive closed-loop control scheme to engineer the fluctuations of the pump field which drives an optomechanical system and show that the corresponding cooling dynamics can be significantly improved. In particular, operating in the counterintuitive "antisquashing" regime of positive feedback and increased field fluctuations, sideband cooling of a nanomechanical membrane within an optical cavity can be improved by $7.5 \mathrm{~dB}$ with respect to the case without feedback. Close to the quantum regime of reduced thermal noise, such feedback-controlled light would allow going well below the quantum backaction cooling limit.
\end{abstract}

DOI: 10.1103/PhysRevLett.119.123603

Feedback loops based on real-time continuous measurements [1] are commonly used for stabilization purposes, and they have also been successfully applied to the stabilization of quantum systems [2-4]. Typically, a system is continuously monitored, and the acquired signal drives the actuator, which, in turn, drives the system to the desired target. Here we demonstrate a novel approach to closedloop control in which the feedback acts on an additional control field which is used to drive the system of interest. In particular, the actuator acts on the control field in order to engineer its phase and amplitude fluctuations. The resulting feedback-controlled in-loop field is then exploited to manipulate the system and improve its performance. Inloop optical fields have been studied for decades both theoretically [5-8] and experimentally [9,10]. A lot of effort has been made to reduce (squash) the noise exhibited by the field fluctuations inside the loop. However, in-loop sub-shot-noise fluctuations cannot be recognized as squeezed below the vacuum noise level, for two different reasons: first, the free field commutation relations are no longer valid for time events separated by more than the loop delay time, since in-loop fields are not free fields [6]; second, the corresponding out-of-loop fields exhibit supershot-noise fluctuations [7]. Nevertheless, useful applications of these fields have been proposed and realized, e.g., suppression of the radiation pressure noise [9], removal of classical intensity noise [10], and atomic line narrowing [8]. The common basis of these works is the negative feedback regime. Negative feedback has also been successfully employed in mechanical [11-13] and cavity optomechanical systems [4], where an electromagnetic field is used to probe a mechanical resonator and, in turn, to control the feedback actuator, which acts directly on the mechanical oscillator. Engineered light fluctuations in the form of squeezed light have also been used in optomechanical systems to improve both the detection sensitivity [14-17] and the cooling efficiency [18-20]. In the present work, we show that it is possible to manipulate, with a feedback system [see Fig. 1(a)], the fluctuations of the laser field that drives an optomechanical system to enhance optomechanical sideband cooling [21-24]. Our analysis demonstrates the effectiveness of this approach in two very different parameter regimes and shows that the light fluctuations can be properly adapted to reduce the effects of the dominant heating processes under very different physical situations. At a low temperature, when standard sideband cooling is limited by backaction noise, we show that the Stokes heating processes can be coherently suppressed by destructive interference so that the quantum backaction limit can be surpassed. At a high temperature, when the performance of sideband cooling is restrained by thermal noise, the feedback can be operated close to instability in order to enhance inelastic light scattering processes and to improve the cooling rate.

A vibrational mode of a mechanical object coupled to a cavity field can be cooled by laser light when the cavity is resonant with anti-Stokes processes, whereby incident photons are scattered to higher frequencies, accompanied by a corresponding reduction in mechanical energy [see Fig. 1(b)]. Residual Stokes processes, instead, heat the mechanical resonator. The rates for Stokes $\left(A_{+}\right)$and antiStokes $\left(A_{-}\right)$processes determine the ultimate efficiency of the cooling process, such that, in the absence of other sources of noise, cooling is constrained by backaction 

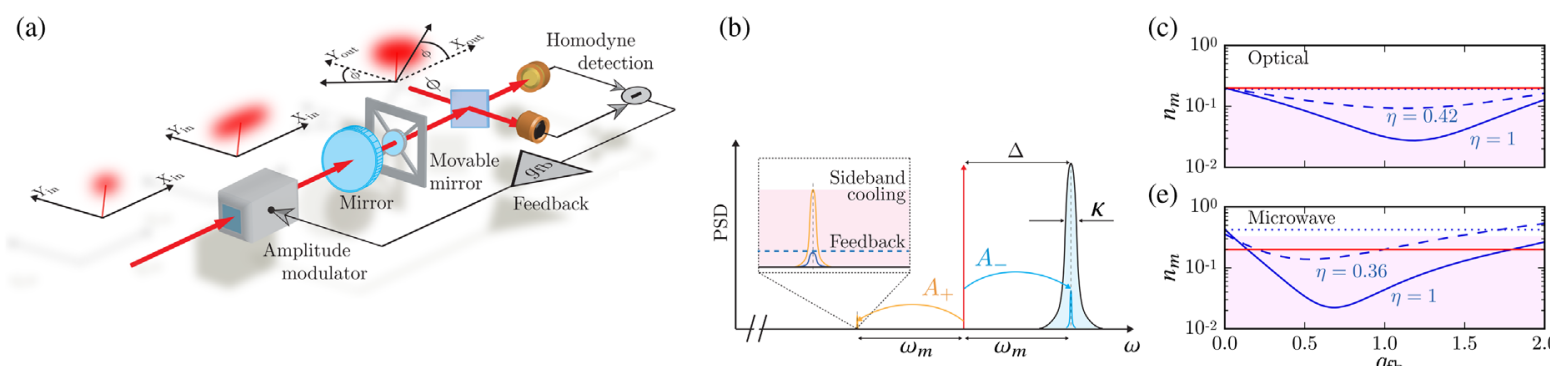

(d)

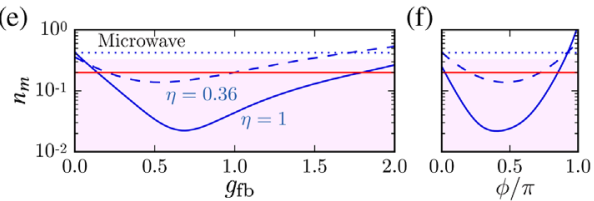

FIG. 1. (a) A cavity is driven by a coherent field with amplitude quadrature $\hat{X}_{\text {in }}$ modified by an amplitude modulator fed with the output of the homodyne quadrature detection. The output field quadratures $\hat{X}_{\text {out }}$ and $\hat{Y}_{\text {out }}$ become correlated, depending on the homodyne phase $\phi$ and on the nonresonant cavity driving at detuning $\Delta$. (b) The radiation pressure interaction in an optomechanical cavity with decay rate $\kappa$ yields sidebands at mechanical frequencies $\pm \omega_{m}$, that correspond to processes which enhance $\left(A_{+}\right.$, yellow) and reduce $\left(A_{-}\right.$, blue) mechanical energy. (c)-(f) Theoretical results for the phonon number of the cooled resonator at a low temperature (when standard sideband cooling is limited by backaction noise), as a function of the feedback gain amplitude $g_{\mathrm{fb}}$ (see [25]) [panels (c) and (e)] and the homodyne phase $\phi$ [panels (d) and (f)], for the parameters of the experiment of Ref. [24] [panels (c) and (d)] and of Ref. [19] [panels (e) and (f)]. The light-pink areas indicate results beyond the backaction limit. Solid blue curves represent results for perfect detection efficiency $\eta=1$, dashed blue curves for $\eta=0.42$ [27] in (c) and (d) and $\eta=0.36$ [28] in (e) and (f), and finally dotted blue curves for no feedback. Red lines are the best up-to-date results obtained for systems operating at the quantum backaction limit, in the optical [24] (with standard sideband cooling) and microwave [19] (where squeezing is employed to improve the system performance) regimes.

noise, which sets the lower limit to mechanical excitations $n_{m}^{0}=A_{+} /\left(A_{-}-A_{+}\right)$. The parameters $A_{+}$and $A_{-}$depend upon the fluctuations of the cavity light, which can be manipulated by enclosing the cooling light in a feedback loop. The feedback operates measuring a generic field quadrature and using the detected signal to modulate the input amplitude quadrature $\hat{X}_{\text {in }}$, while the conjugate phase quadrature $\hat{Y}_{\text {in }}$ remains untouched. In particular, $\hat{X}_{\text {in }}$ is modified according to the feedback relation

$\hat{X}_{\mathrm{in}} \rightarrow \frac{1}{1-2 g_{\mathrm{fb}}(\omega) \zeta_{\mathrm{out}}^{(\phi)}(\omega)}\left[\hat{X}_{\mathrm{in}}+2 g_{\mathrm{fb}}(\omega) \zeta_{\mathrm{out}}^{(\phi+\pi / 2)}(\omega) \hat{Y}_{\mathrm{in}}\right]$

where $g_{\mathrm{fb}}(\omega)$ is the electronic feedback transfer function and $\zeta_{\text {out }}^{(\phi)}(\omega)$ and $\zeta_{\text {out }}^{(\phi+\pi / 2)}(\omega)$, defined in Supplemental Material [25], describe the response of the output field to the input amplitude and phase fluctuations, respectively; i.e., in the absence of feedback, the detected output field quadrature is $\hat{X}_{\text {out }}^{(\phi)}=\zeta_{\text {out }}^{(\phi)}(\omega) \hat{X}_{\text {in }}+\zeta_{\text {out }}^{(\phi+\pi / 2)}(\omega) \hat{Y}_{\text {in }}$, and additional noise terms not relevant to the discussion are omitted both in Eq. (1) and in the inline equation [25].

The corresponding mechanical scattering rates [25]

$$
A_{ \pm}=\frac{G^{2}}{2 \kappa}\left|\chi_{c}\left(\mp \omega_{m}\right)+\left(\frac{2 g_{\mathrm{fb}}\left(\mp \omega_{m}\right) \zeta_{c}^{(0)}\left(\mp \omega_{m}\right)}{1-2 g_{\mathrm{fb}}\left(\mp \omega_{m}\right) \zeta_{\text {out }}^{(\phi)}\left(\mp \omega_{m}\right)} e^{i \phi}\right)^{*}\right|^{2}
$$

are proportional to the square of the optomechanical coupling strength $G$ and are given by the superposition of two contributions. The first term is related to the standard sideband laser cooling, which can be expressed in terms of the susceptibility $\chi_{c}(\omega)=2 \kappa /[\kappa+i(\Delta-\omega)]$ of a cavity with linewidth $\kappa$ and detuning $\Delta$. The second term is instead determined by the feedback loop. The function $\zeta_{c}^{(0)}(\omega)$ describes the response of the cavity field amplitude $\hat{X}$ to the input amplitude fluctuations; i.e., in the absence of feedback it can be expressed in terms of the input field as $\sqrt{2 \kappa} \hat{X}=\zeta_{c}^{(0)}(\omega) \hat{X}_{\text {in }}+\zeta_{c}^{(\pi / 2)}(\omega) \hat{Y}_{\text {in }}$ (once more, additional noise terms are omitted). It is important to note that the feedback term sums up coherently and can be properly optimized to enhance the performance of sideband cooling. Specifically, Stokes processes can be fully suppressed, $A_{+}=0$ (and therefore the backaction limit is surpassed), when the cavity and feedback contributions interfere destructively and cancel each other, which is achieved by setting the feedback gain value to $2 g_{\mathrm{fb}}\left(-\omega_{m}\right)=$ $\chi_{c}\left(-\omega_{m}\right)^{*} /\left[\zeta_{\text {out }}^{(\phi)}\left(-\omega_{m}\right)-\zeta_{c}^{(0)}\left(-\omega_{m}\right) e^{i \phi}\right]$. So far, we have assumed perfect detection efficiency, meaning that all the light lost by the cavity is detected and employed in the loop. In practice, at finite detection efficiencies, Stokes processes cannot be fully suppressed. Nevertheless, also in realistic cases a strong reduction of $A_{+}$is observed, and this approach can outperform the best up-to-date results obtained for systems operating at the quantum backaction limit, both in the optical [24] and in the microwave [19] regime, as shown in Figs. 1(c)-(f). These results correspond to situations in which thermal noise is so low that standard sideband cooling is essentially limited by backaction noise. In general, thermal fluctuations, characterized by the number of thermal excitations $n_{m}^{\text {th }}$, compete with the effect of the cooling light to determine the stationary phonon occupancy $n_{m}=\left(\gamma_{m} n_{m}^{\text {th }}+\Gamma_{\text {opt }} n_{m}^{0}\right) /\left(\gamma_{m}+\Gamma_{\text {opt }}\right)$, 

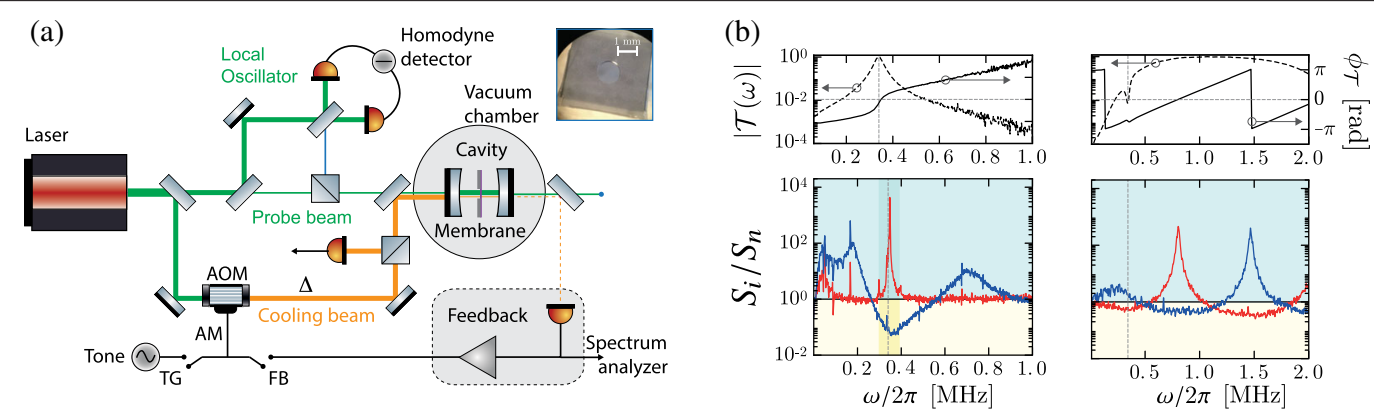

FIG. 2. (a) Optomechanical cavity driven by the in-loop cavity mode (cooling beam). Dynamical backaction of the fundamental mechanical mode is provided by detuning the cooling beam by means of an AOM. Feedback is applied by AM the cooling laser with an electronically processed copy of the transmitted photocurrent. Inset: Image of the circular SiN membrane used, radius 0.615 mm and thickness $97 \mathrm{~nm}$. The switches TG and FB allow the open- and closed-loop transfer functions to be measured [25]. A probe beam is used to monitor the cavity frequency fluctuations via balanced homodyne detection. (b) Current noise spectra $S_{i}$, normalized to the detection noise $S_{n}$, of the transmitted (bottom left) and reflected (bottom right) photocurrent measured placing the membrane in a position of zero optomechanical interaction $g_{0}=0$. Red and blue traces correspond to different signs of the amplifier output in the electronic filter. The vertical dashed gray line indicates the detuning $\Delta=2 \pi \times 330 \mathrm{kHz}$. The light-blue (light-yellow) area represents the antisquashing (squashing) regime, where noise is amplified (reduced) below the detection noise. We refer to the gain for which the feedback based on transmission provides antisquashing around the cavity detuning (shaded area) as positive. The top part shows the corresponding measured amplitude (dashed lines) and phase (solid lines) of the complex open-loop feedback response function $\mathcal{T}(\omega)$ determined from the transmitted light detected with the switch FB open [25].

where $\gamma_{m}$ and $\Gamma_{\text {opt }}=\left(A_{-}-A_{+}\right)$are the mechanical and optical damping rates, respectively. Hence, at a high temperature, aiming at barely suppressing Stokes processes becomes ineffective. However, in this regime, the effects of thermal noise can be strongly reduced by operating the feedback close to instability such that $\Gamma_{\text {opt }}$ is increased to large values, at the expense of increasing the backaction limit $n_{m}^{0}$

We have tested this high-temperature regime with a double-sided, 90-mm-long symmetric cavity [34,35], with a decay rate $\kappa=2 \pi \times 20.15 \mathrm{kHz}$. A SiN membrane is placed in the middle of the optical cavity $[34,36]$. It is a highly stressed circular membrane, with a diameter of $1.2 \mathrm{~mm}$, a thickness of $97 \mathrm{~nm}$, and negligible optical absorption [26]. We focus on the fundamental mechanical mode, characterized by a resonance frequency $\omega_{m}=$ $2 \pi \times 343.13 \mathrm{kHz}$ and a decay rate $\gamma_{m}=2 \pi \times 1.18 \mathrm{~Hz}$. The optomechanical coupling is $G=g_{0} \sqrt{2 n_{c}}$, with $n_{c}$ the number of cavity photons and $g_{0}$ the single-photon coupling, tunable by translating the membrane within the cavity standing wave [25]. Two beams, the probe (green lines) and the cooling beam (orange lines), are derived from a $1064 \mathrm{~nm}$ master laser (see Fig. 2). The former, which is not part of the feedback loop, is used to lock the laser frequency to the cavity resonance and to reveal the mechanical displacement by homodyne detection. The cooling beam, detuned from the relevant cavity resonance by $\Delta=2 \pi \times 330 \mathrm{kHz}$, is, instead, enclosed in the feedback loop. The amplitude quadrature (corresponding to $\phi=0$ ) of the transmitted (reflected) field is directly detected with a single photodiode, and the resulting photocurrent, eventually filtered and amplified [in Fig. 2(a), the filter is applied to the transmitted light], is fed back to the input field by amplitude modulating (AM) the acousto-optic modulator (AOM) [25].

We first measure the in-loop light properties by placing the membrane at a node of the cavity field to rule out the optomechanical interaction. The feedback loop is fully characterized by measuring the open-loop transfer function $\mathcal{T}(\omega)$, which includes both the electronic and the optical response of the system [see Fig. 2(b), top, and Ref. [25]]. The electronic part, $g_{\mathrm{fb}}(\omega)$, is generally complex due to the feedback delay time $\tau_{\mathrm{fb}}$, which, in our case, is $750 \mathrm{~ns}$. When the feedback loop is closed, the amplitude noise fluctuations are modified, as shown in Fig. 2(b), bottom; the noise becomes frequency dependent, with regions below (noise squashing) and above (noise antisquashing) the noise level with no feedback [6,7]. The feedback-controlled cavity also shows a modified susceptibility in the antisquashing regime, as can be verified by sending a weak classical seed field, larger than all noises but too small to affect the mean cavity amplitude. In the regime of our system, $\Delta \gg \kappa$ and small delay time $1 / \tau_{\mathrm{fb}} \gg \kappa$ (so that a single antisquashing resonance [see Fig. 2(b)] contributes to the dynamics), and for frequencies close to the cavity resonance, the seed experiences an effective cavity susceptibility $\chi_{c}^{\text {eff }}(\omega)=2 \kappa\left[\kappa_{\text {eff }}+i\left(\Delta_{\text {eff }}-\omega\right)\right]^{-1}$, with $\kappa_{\text {eff }}=\kappa\left(1-\mathcal{G}_{\mathrm{fb}}\right)$ and $\Delta_{\text {eff }}=\Delta-\kappa \mathcal{G}_{\mathrm{fb}} \tan \left[\phi_{\mathcal{T}}(\Delta)\right]$, where $\mathcal{G}_{\mathrm{fb}}$ is the normalized feedback gain, which is $\mathcal{G}_{\mathrm{fb}}=1$ at the feedback stability threshold defined by $\kappa_{\text {eff }}=0$, and $\phi_{\mathcal{T}}(\Delta)$ the phase of the feedback response function $\mathcal{T}(\omega)$ at the detuning $\Delta[25]$. Experimentally, we determine $\chi_{c}^{\text {eff }}(\omega)$ by measuring the closed-loop transfer function for different feedback gains (see Fig. 3). By increasing the gain, the system approaches the feedback stability threshold; i.e., $\kappa_{\text {eff }}$ tends to 0, as shown in Figs. 3(c) and 3(d). We were able to 

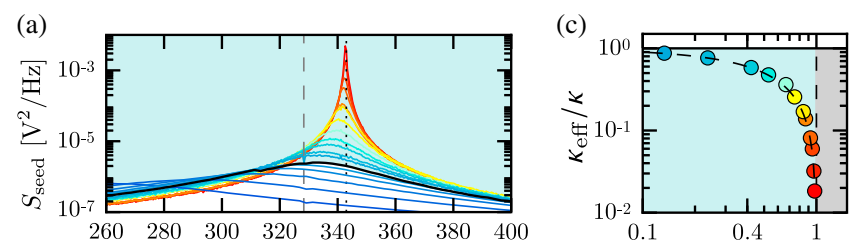

(b)
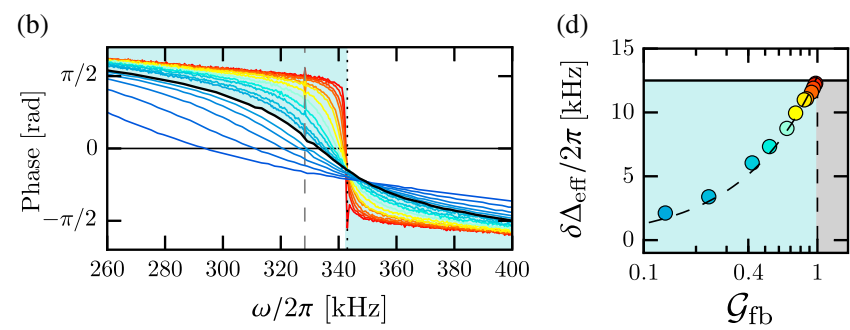

FIG. 3. (a) Amplitude and (b) phase of the measured closedloop transfer function for the transmitted cooling field detuned by $\Delta=2 \pi \times 330 \mathrm{kHz}$ (dashed gray line) [25]. Black traces are acquired without the feedback loop. The dotted black line indicates the effective cavity frequency at instability. (c) Effective cavity decay rate $\kappa_{\text {eff }}$, normalized to the out-of-loop decay rate $\kappa$, and (d) effective detuning shift $\delta \Delta_{\text {eff }} \equiv \Delta_{\text {eff }}-\Delta$ as a function of the positive feedback gain. For positive feedback (from light blue to red) the effective cavity decay rate $\kappa_{\text {eff }}$ decreases and the effective detuning $\Delta_{\text {eff }}$ increases, while for negative feedback (from light to dark blue) the situation is reversed. The gray area represents the instability region.

reach a minimum effective cavity linewidth of $\kappa_{\text {eff }} \approx 2 \pi \times$ $250 \mathrm{~Hz}$ and a detuning of $\Delta_{\text {eff }} \approx 2 \pi \times 342.5 \mathrm{kHz}$.

So far, we have characterized the feedback system with $g_{0}=0$, and we have determined the properties of the cavity experienced by the resonator which do not depend on the optomechanical interaction (i.e., the values of $\kappa_{\text {eff }}$ and $\Delta_{\text {eff }}$ ). These measurements are sufficient to perfectly reproduce the experimental cooling results reported below. The positive feedback regime (antisquashing), which we focus on, enhances light amplitude noise. We show that driving an optomechanical cavity with the resulting in-loop field improves cooling by increasing the optical cooling rate. The membrane is now placed in a position in which $g_{0}=$ $2 \pi \times 0.84 \mathrm{~Hz}$ [25]. Without feedback, a beam of $33 \mu \mathrm{W}$ red detuned by $\Delta=2 \pi \times 330 \mathrm{kHz}$ [see Fig. 4(a)] cools the membrane by dynamical backaction [21] from room temperature to an effective temperature of $2 \mathrm{~K}$. As the transmission feedback loop is closed and the gain is varied, the mechanical susceptibility, determined with the out-ofloop probe field, is shifted and broadened, as seen from Fig. 4(a) and Ref. [25]. The corresponding reduction of the effective mechanical energy reported in Fig. 4(b) in terms of the number of mechanical excitations $n_{m}$, and computed by numerical integration of the spectra [25], demonstrates an enhancement of the cooling rate, which settles the minimum effective temperature to $\hbar \omega_{m} n_{m} / k_{B}=350 \mathrm{mK}$ for an optimal gain of $\mathcal{G}_{\mathrm{fb}} \sim 0.9$. Having fixed the optimal gain, we measured the effective mechanical energy as a
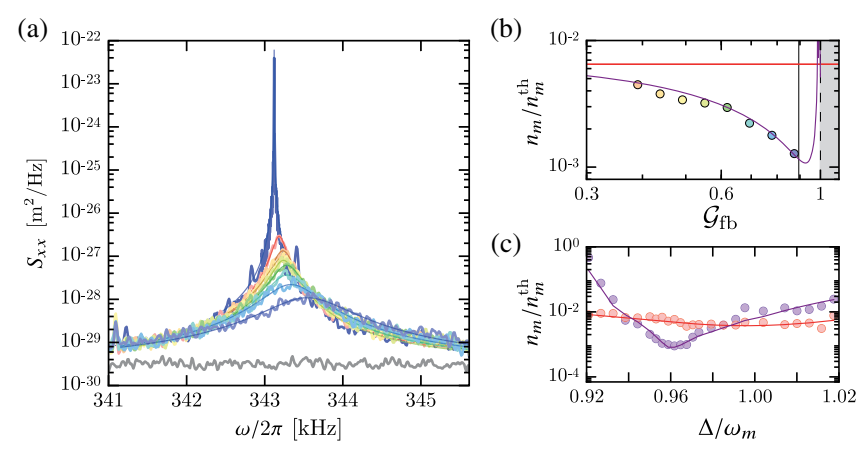

FIG. 4. (a) Homodyne spectra of mechanical displacement noise $S_{x x}$. The blue trace represents the thermal fluctuations of the fundamental mechanical mode at $300 \mathrm{~K}$; the gray trace is the detection noise. Dynamical backaction cools the mechanical motion down to $2 \mathrm{~K}$, as shown by the red trace (cooling beam on at $33 \mu \mathrm{W}$ and feedback off). From orange to light purple, the feedback is turned on and the gain increased. (b),(c) Effective energy reduction as a function of the gain $\left(\mathcal{G}_{\mathrm{fb}}\right)$ and normalized detuning $\left(\Delta / \omega_{m}\right)$ [25]. Dots are experimental data. Each dot in (b) corresponds to the spectrum of the same color in (a), the gray area represents the instability region, and the vertical gray line indicates the optimal gain value for cooling, used in plot (c). In (c), purple and red dots are results with and without feedback, respectively. In both panels, purple and red lines are theoretical results, computed using the measured parameters, with and without feedback, respectively.

function of the detuning [Fig. 4(c)], reaching the minimum phonon number for the optimal bare detuning $\Delta^{\mathrm{opt}}=$ $2 \pi \times 329.4 \mathrm{kHz}$. This value is consistent with the one estimated by using the measured feedback phase margin at the detuning frequency, $\phi_{\mathcal{T}}\left(\Delta^{\mathrm{opt}}\right) \sim-0.59 \mathrm{rad}$, and by setting the feedback gain at instability, $\mathcal{G}_{\mathrm{fb}}=1$, and the effective detuning at the optimal resolved sideband cooling condition $\Delta_{\text {eff }} \sim \omega_{m}$, that is, $\Delta^{\text {opt }} \sim \omega_{m}+\kappa \tan \left[\phi_{\mathcal{T}}\left(\Delta^{\text {opt }}\right)\right]$.

Our results demonstrate that the in-loop field fluctuations obtained in the counterintuitive regime of positive feedback can be exploited for enhancing the cooling efficiency in an optomechanical system. In the regime of low thermal noise, we find theoretically that our approach allows the backaction limit to be beaten by almost an order of magnitude, in both the optical and the microwave regime [see Figs. 1(c)-1(f)], as a result of the engineered intracavity field fluctuations, which contribute to the coherent cancellation of Stokes processes and hence to the reduction of the backaction limit. This result is analogous to that discussed in Refs. [19,20], which make use of squeezed light, but is achieved with a significantly simpler setup which does not require quantum nonlinearities. The foreseen dynamics should be observable including the feedback system in, for example, the experimental setup of Refs. [19,24]. This would require using homodyne detection, with a properly optimized phase of the detected quadrature, and sufficiently large detection efficiency. With our setup, which instead does not work at cryogenic 
temperature, we operate the feedback close to the instability and achieve an enhancement of the cooling rate of $10 \mathrm{~dB}$, with a corresponding reduction of the phonon number of $7.5 \mathrm{~dB}$ (see Fig. 4). In this limit, the effectiveness of the feedback is associated with a reduction of the cavity linewidth, which increases the optomechanical cooperativity. The fact that the enhancement of the cooling rate is not reflected in an equal reduction of the number of mechanical excitations is due to the concomitant increase of the backaction limit in this high-temperature regime, which prevents further cooling of the resonator [25]. The generic technique that we have demonstrated can be adopted in a broad range of applications, whenever a system of interest is controlled with an electromagnetic field subject to a phase-sensitive measurement, in the classical as well as the quantum regime.

We acknowledge the support of the European Union's Horizon 2020 research and innovation program under grant agreement No. 732894 (FET Proactive HOT).

*gianni.digiuseppe@unicam.it david.vitali@unicam.it

[1] H. M. Wiseman and G. J. Milburn, Quantum Measurement and Control (Cambridge University Press, Cambridge, England, 2010).

[2] C. Sayrin et al., Nature (London) 477, 73 (2011).

[3] D. Ristè, M. Dukalski, C. A. Watson, G. de Lange, M. J. Tiggelman, Y. M. Blanter, K. W. Lehnert, R. N. Schouten, and L. DiCarlo, Nature (London) 502, 350 (2013).

[4] D. J. Wilson, V. Sudhir, N. Piro, R. Schilling, A. Ghadimi, and T. J. Kippenberg, Nature (London) 524, 325 (2015).

[5] M. S. Taubman, H. Wiseman, D. E. McClelland, and H.-A. Bachor, J. Opt. Soc. Am. B 12, 1792 (1995).

[6] H. M. Wiseman, J. Opt. B 1, 459 (1999).

[7] J. H. Shapiro, G. Saplakoglu, S.-T. Ho, P. Kumar, B. E. A. Saleh, and M. C. Teich, J. Opt. Soc. Am. B 4, 1604 (1987).

[8] H. M. Wiseman, Phys. Rev. Lett. 81, 3840 (1998).

[9] B. C. Buchler, M. B. Gray, D. A. Shaddock, T. C. Ralph, and D. E. McClelland, Opt. Lett. 24, 259 (1999).

[10] B. S. Sheard, M. B. Gray, B. J. J. Slagmolen, J. H. Chow, and D. E. McClelland, IEEE J. Quantum Electron. 41, 434 (2005).

[11] P. F. Cohadon, A. Heidmann, and M. Pinard, Phys. Rev. Lett. 83, 3174 (1999).

[12] M. Poggio, C. L. Degen, H. J. Mamin, and D. Rugar, Phys. Rev. Lett. 99, 017201 (2007).

[13] A. Vinante et al., Phys. Rev. Lett. 101, 033601 (2008).

[14] K. McKenzie, D. A. Shaddock, D. E. McClelland, B. C. Buchler, and P. K. Lam, Phys. Rev. Lett. 88, 231102 (2002).

[15] J. Aasi et al., Nat. Photonics 7, 613 (2013).
[16] V. Peano, H. G. L. Schwefel, C. Marquardt, and F. Marquardt, Phys. Rev. Lett. 115, 243603 (2015).

[17] J. B. Clark, F. Lecocq, R. W. Simmonds, J. Aumentado, and J. D. Teufel, Nat. Phys. 12, 683 (2016).

[18] C. Schäfermeier, H. Kerdoncuff, U. B. Hoff, H. Fu, A. Huck, J. Bilek, G. I. Harris, W. P. Bowen, T. Gehring, and U. L. Andersen, Nat. Commun. 7, 13628 (2016).

[19] J. B. Clark, F. Lecocq, R. W. Simmonds, J. Aumentado, and J. D. Teufel, Nature (London) 541, 191 (2017).

[20] M. Asjad, S. Zippilli, and D. Vitali, Phys. Rev. A 94, 051801 (2016).

[21] M. Aspelmeyer, T. J. Kippenberg, and F. Marquardt, Rev. Mod. Phys. 86, 1391 (2014).

[22] J. D. Teufel, T. Donner, D. Li, J. W. Harlow, M. S. Allman, K. Cicak, A. J. Sirois, J. D. Whittaker, K. W. Lehnert, and R. W. Simmonds, Nature (London) 475, 359 (2011).

[23] J. Chan, T. P. Mayer Alegre, A. H. Safavi-Naeini, J. T. Hill, A. Krause, S. Gröblacher, M. Aspelmeyer, and O. Painter, Nature (London) 478, 89 (2011).

[24] R. W. Peterson, T. P. Purdy, N. S. Kampel, R. W. Andrews, P.-L. Yu, K. W. Lehnert, and C. A. Regal, Phys. Rev. Lett. 116, 063601 (2016).

[25] See Supplemental Material at http://link.aps.org/ supplemental/10.1103/PhysRevLett.119.123603 for a comprehensive description of the mathematical model at the basis of our investigation and for details regarding the experimental setup and the analysis of the experimental data, which includes Refs. [19,24,26-33].

[26] E. Serra et al., AIP Adv. 6, 065004 (2016).

[27] T. P. Purdy, P.-L. Yu, R. W. Peterson, N. S. Kampel, and C. A. Regal, Phys. Rev. X 3, 031012 (2013).

[28] F. Mallet, M. A. Castellanos-Beltran, H. S. Ku, S. Glancy, E. Knill, K. D. Irwin, G. C. Hilton, L. R. Vale, and K. W. Lehnert, Phys. Rev. Lett. 106, 220502 (2011).

[29] C. Genes, D. Vitali, P. Tombesi, S. Gigan, and M. Aspelmeyer, Phys. Rev. A 77, 033804 (2008).

[30] E. D. Black, Am. J. Phys. 69, 79 (2001).

[31] H. P. Yuen and V. W. S. Chan, Opt. Lett. 8, 177 (1983).

[32] M. L. Gorodetsky, A. Schliesser, G. Anetsberger, S. Deleglise, and T. J. Kippenberg, Opt. Express 18, 23236 (2010).

[33] W. C. Elmore and M. A. Heald, Physics of Waves (Dover, New York, 1985).

[34] M. Karuza, C. Molinelli, M. Galassi, C. Biancofiore, R. Natali, P. Tombesi, G. Di Giuseppe, and D. Vitali, New J. Phys. 14, 095015 (2013).

[35] M. Karuza, C. Biancofiore, M. Bawaj, C. Molinelli, M. Galassi, R. Natali, P. Tombesi, G. Di Giuseppe, and D. Vitali, Phys. Rev. A 88, 013804 (2013).

[36] J. D. Thompson, B. M. Zwickl, A. M. Jayich, F. Marquardt, S. M. Girvin, and J. G. E. Harris, Nature (London) 452, 72 (2008). 